DOE/BC/14600-9

Distribution Category UC-122

\title{
PERCOLATION MODELS FOR BOILING AND BUBBLE GROWTH IN POROUS MEDIA
}

\author{
Topical Report \\ $\mathrm{DOE} / \mathrm{BC} / 14600--9$ \\ By \\ DE91 002241 \\ Yanis C. Yortsos
}

May 1991

Work Performed Under Contract No. DE-FG22-90BC14600

Prepared for

U.S. Department of Energy

Assistant Secretary for Fossil Energy

Thomas B. Reid, Project Manager

Bartlesville Project Office

P. O. Box 1398

Bartlesville, OK 74005

Prepared by

University of Southern California

University Park

Los Angeles, CA 90089-1211

MASTER

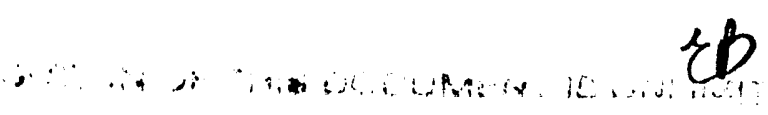




\section{Contents}

1 ABSTRACT 1

2 INTRODUCTION 2

3 PHASE EQUILIBRIA IN POROUS MEDIA 3

4 NUCLEATION 5

$\begin{array}{lll}5 & \text { BUBBLE GROWTH } & 7\end{array}$

5.1 Dimensionless Parameters $\ldots \ldots \ldots \ldots \ldots \ldots \ldots$

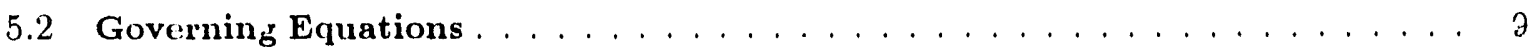

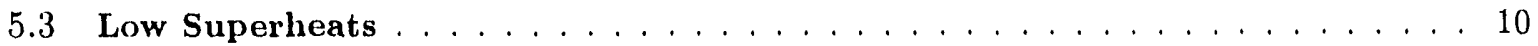

5.4 Moderate and Large Superheats $\ldots \ldots \ldots \ldots \ldots \ldots \ldots$

6 PERCOLATION MODELS 12

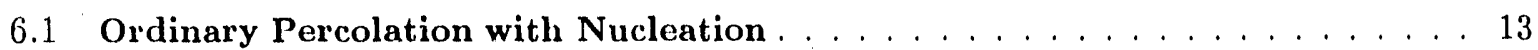

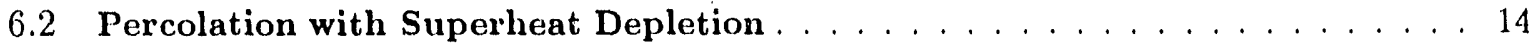

6.3 Gradient Percolation with Nucleation $\ldots \ldots \ldots \ldots \ldots \ldots \ldots$

7 CONCLUSIONS 16

8 ACKNOWLEDGEMENTS 17

9 REFERENCES 18 


\section{List of Tables}

1 Typical Values of Parameters. . . . . . . . . . . . . . 20

\section{List of Figures}

1 Schematic of a "Bubble" in a Porous Medium. . . . . . . . . . . . . . . . . 20

2 Schematic of Equilibrium and Supersaturations. . . . . . . . . . . 21

3 Stability of a Vapor-Liquid Interface in a Converging Geometry. . . . . . . . . . 22

4 Schematic of a Nucleation Site in a Pore Body. . . . . . . . . . . . . 23

5 Bubble Radius-Volume Equilibrium for the Hydrophobic Cavity of Fig. 4. . . . . 24

6 Bubble Growth in Percolation from Nucleation Sites in a Square Lattice at Three Different Stages of Growth $(p=0.221,0.555$ and 0.9988 , respectively) . . . . . 25

7 Relative Permeabilities as a Function of $\beta$ (from [14]) . . . . . . . . . . 26

8 Critical Gas Saturation as a Function of $\beta$ (from [14], $\kappa=0.1) \ldots \ldots . \ldots 27$

9 Supersaturation Paths for Adiabatic Depletion: (a) Pressure, (b) Temperature. . 28

10 Vapor-Liquid Equilibrium in a Temperature Gradient Using Gradient Percolation for Different Values of $\beta$ : (a) $\beta=0.2$, (b) $\beta=0.5 \ldots \ldots \ldots$. . . . . . 29 


\section{PERCOLATION MODELS FOR BOILING AND BUBBLE GROWTH IN POROUS MEDIA. AT LOW SUPERHEATS}

by

C. Satik and Y. C. Yortsos

\section{ABSTRACT}

We analyze the liquid-to-vapor phase change in single-component fluids in porous media at low superheats. Conditions typical to steam injection in porous media are taken. We examine nucleation, phase equilibria and their stability, and the growth of vapor bubbles. Effects of pore structure are emphasized. It is shown that at low supersaturations, bubble growth can be described as a percolation process. In the absence of spatial gradients, macroscopic flow properties are calculated in terms of nucleation parameters. A modification of gradient percolation is also proposed in the case of spatial temperature gradients, when solid conduction predominates. 


\section{INTRODUCTION}

The liquid-to-vapor phase change in porous media is a process fundamental to many phenomena, such as drying and boiling, and it is common to diverse applications, such as geothermal, enhanced oil recovery and nuclear waste disposal. Despite its demonstrated significance, howcver, it is at present poorly understood. With few exceptions, most of the a ailable studies are of a phenomenological nature, relying on the conventional extension of Darcy's law and on the use of relative permeability and capillary pressure functions, typically borrowed from the literature on gas-liquid displacement. Examples abound in the respective applications [1-4]. Lacking, in particular, is the understanding of the interplay between phase equilibria and phase change, the degree and rate of change of supersaturation, the heat transfer and the pore structure. The latter introduces aspects different than for phase change in the bulk [5-7]. This is especially true for porous media of moderate-to-low permeability, where capillary phenomena (an be significant.

Although recent publications in the Russian literature peripherally touch on some of these issues [8-10], concerted efforts to address various problems were made by Udell [11], who attempted to describe vapor-liquid equilibria in porous media, and by Parlar and Yortsos [12], who proposed a percolation model for steam-water relative permeabilities. Both approaches were incomplete, however, in that the first largely ignored the pore structure, while the second neglected any heat transfer or supersaturation considerations. It must be pointed out that the model of Parlar and Yortsos (1987) for drainage did indeed corroborate the experimental findings of Sanchez and Schechter (1987) for concurrent flow. Nevertheless, additional work is necessary to support their findings and to describe the process for other flow configurations. Recently, Yortsos and Parlar [14] made significant advances on the related problem of solution gas-drive. The system considered was two-component, two-phase, with the bubble growth controlled by solubility, pressure decline and mass transfer. The authors considered various aspects of the process including phase equilibria and nucleation, and proposed a percolation model for bubble growth from many nucleation sites.

The work in [14] is the first to analyze systematically the bubble growth process, although specific solutions were derived only for rather special cases. Since the two problems share many common aspects we can apply a similar approach to describe the phase change and the 
subsequent bubble growth of a single-component system driven by an imposed superheat. This forms the main objective of this paper. In the context of boiling in porous media, we will address equilibria of vapor-liquid interfaces, issues of heterogeneous nucleation, the subsequent bubble growth and effects of superheat and heat transfer. The analysis is intended to probe the validity of the conventional approach, to derive constraints on its applicability and to illustrate growth patterns. A key element in our models is the representation of the porous medium in terms of a connected (but uncorrelated) network of pore throats and pore bodies of variable size. With the assumption of lack of spatial correlations, we are in a position to take advantage of novel statistical tools, such as percolation theory, to describe the growth process. This allows us to demonstrate clearly the difference between bubble growth in a porous medium and that in the bulk.

Although a general analysis is attempted, specific results are obtained only for the case of low superheats (slow growth). Under such conditions, expected to be valid for steam injection processes in oil recovery or for geothermal reservoirs, temperature and pressure gradients are small, thus relative permeabilities are only saturation (and history) dependent. As will become apparent, nucleation effects significantly influence the macroscopic properties. We will illustrate two applications, one in which spatial temperature gradients are negligible and the heating is uniform, and another in which heat conduction through the solid matrix dominates the distribution of phases. Although progress is made in both directions, significant work still remains to be done, as will be pointed out below.

\section{PHASE EQUILIBRIA IN POROUS MEDIA}

We consider a vapor bubble in equilibrium with a liquid in a porous medium (Figure 1). We assume that nucleation has ceased and that individual interface menisci in pores are spherical (although macroscopic shapes are certainly not spherical). At conditions of equilibrium, temperature, liquid and vapor pressures are all spatially uniform. Thermodynamics dictate that the Laplace equation is satisfied, thus relating fluid pressures to the radius of curvature of any vapor-liçuid meniscus

$$
P_{V}-P_{L}=\frac{2 \gamma}{r}
$$

where $\gamma$ is the surface tension. For simplicity, we neglert Kelvin effects and assume that the pressure in the vapor phase, $P_{V}$, is equal to the saturation vapor pressure at $T_{L}$, e.g., as given 
by the Clausius-Clapeyron equation

$$
P_{V}=P_{V O}\left(T_{L}\right)
$$

It is worth pointing out that the importance of Kelvin effects has been exaggerated in the previous literature (e.g. [11,12]). In a recent study [15], using a conventional macroscopic description we have shown that, except for very tight porous media (with permeability on the order of microdarcies), or for very low liquid saturation values, Kelvin effects are generally of secondary significance.

Phase change and phase equilibria in a porous medium may be schematically interpreted as in Figure 2. The system, initially in the liquid state (point A), is slowly heated at constant pressure, until the equilibrium curve is reached (point B). Further increase in temperature leads to the superheat $\Delta T=T_{L}-T_{\text {sat }}$, where $T_{s a t}$ is the saturation temperature at, $P_{L}$. In a liquid in the bulk, the appearance of the first bubble theoretically occurs at the onset of homogenous nucleation, which requires substantial amounts of superheat. In a porous medium, on the other hand, the onset of phase change is facilitated by heterogeneous nucleation. Given that a bubble has formed (point $\mathrm{C}$ ), the pressure in the vapor phase at equilibrium is given by (2). Mechanical equilibrium of the vapor-liquid interfaces in the porous media "bubble" (Figure 1) requires spherical shape menisci of constant mean curvature $H_{0}$

$$
P_{V O}\left(T_{L}\right)-P_{L}=2 g H_{0}
$$

The RHS in the above is a measure of the capillary pressure and it is set by the porous medium.

Contrary to processes in the bulk, where unbounded growth ensues, pore walls in porous media stabilize interfaces, thus vapor-liquid equilibria are possible. The stability of gas-liquid interfaces in porous media for two-component, two-phase systems was summarized by Hirasaki et. al. [16]. It was shown that interfaces residing on converging pore geometries are stable, while these on diverging geometries are unstable. Yortsos and Parlar (1989) extended their analysis to account for mass transfer by diffusion in the case of two-component, vapor-liquid systems. They found that the stability of menisci on converging pore geometries is enhanced by mass transfer. Analogous results should apply for the system under consideration here. In the following, we adapt the qualitative part of their argument to show equivalent effects for the single-component, vapor-liquid system of interest to boiling. 'The mathematical details are 
similar, but generally complex, and we refer the reader to Appendix A of [14].

Consider a small perturbation in the curvature of a spherical, vapor-liquid interface located in a converging pore element (from pore body-to-pore throat, Figure 3). For this interface to advance, the mean cuvature must increase. For a constant liquid pressure, this requires an increase in the vapor pressure, or equivalently, in the interface temperature. This increase sets, in turn, a temperature gradient, and a concommitant heat flux away from the vapor towards the liquid, which results in condensation, thus in a retraction of the interface to its original position. An equivalent argument applies if the initial perturbation is in the oposite direction. One is led to conclude that the equilibrium of a vapor-liquid meniscus in a conveging pore geometry is additionally stabilized by heat transfer and phase equilibria. This is not the case for a diverging geometry, where stable equilibria are not possible, much like the case of vapor bubbles in the bulk. The existence of stable interfaces in pore geometries is fundamental to heterogeneous nucleation, an issue to which we turn next.

\section{NUGLEATION}

Nucleation processes in porous media are quite complex and there are still several questions that remain unanswered. However, some reasonable progress has been made. It is evident that in the majority of cases we can discard homogeneous nucleation as a plausible mechanism because of its restrictive conditions of perfectly smooth and liquid wet solid surfaces, the absence of trapped gases, and the unrealistically high supersaturations (of the order of several thousand psi for the solution gas-drive example of Yortsos and Parlar (1989)). All these conditions are not likely to be met in many instances. Instead, heterogeneous nucleation is the mechanism likely to dominate the phase change process.

Heterogeneous nucleation has been analyzed by Yortsos and Parlar (1989), who proposed a conventional nucleation model. It is based on the assumption that there exist pore surface irregularities (cracks, scratches, pits), which are not liquid wet and which contain pre-existing or trapped gases, that act as nucleation sites. For convenience in calculations, the sites were assumed of a conical shape (Figure 4). Because of the premise of heterogeneous mechanism, the issue of nucleation rates does not arise. Instead, sites are activated and a pore body is allowed to be occupied by vapor when the vapor-liquid meniscus in the cavity loses stability. As shown below, this occurs when the interface reaches the cavity mouth. 
To examine the nucleation mechanism, we consider a hydrophobic conical cavity and evalwate the variation of the radius of curvature of the liequid-vapor interface with the volume of the vapor (Figure 5). Calculations of such configurations were made by Forrest (1982). As previously pointed out, stable interfaces correspond to configurations such that $d r / d v<0$, i.e. the radius of curvature decreases with an increase in the volume of the gas phase. Note that a negative radius of curvature implies an interface concave to the liquid phase. From the schematic in Figure 5 it follows that until the radius $r$ reaches the pit mouth (and $r=W$ ), the equilibrium state is stable, suggesting that the vapor is confined in the cavity. When the cavity mouth is reached, however, a further increase in the supersaturation (superheat) renders the equalibrim state unstable. The bubble would grow in a non-equilibrium fashion, much like in the bulk, until its radius of curvature equals the host pore body radius $r_{s}$. Due to the pore wall constraints, further increase in the volume causes a decrease in the radius of curvature. This step is also stable, although it may not necessarily be an equilibrium one at the particular supersaturation (its radius may be larger than $W$ ), in which case the bubble may further grow.

By contrast, a vapor bubble in the bulk liquid is in unstable mechanical equilibrium, that enforces continuous growth or collapse. The fact that due to pore wall stabilization, a vapor bubble can exist in stable equilibrium with a superheated liquid, is a major difference between porous media and bulk. With $W$ denoting the cavity mouth radius, we conclude that the onset of nucleation of a site occurs when

$$
P_{V O}\left(T_{L}\right)-P_{L}=\frac{2 \gamma}{W}
$$

To express this condition in terms of the host pore body radius, we adopt from Yortsos and Parlar (1989) the geometric factor $\beta=W / r_{s}$, which is the ratio of cavity mouth radius to pore body radius. Given the latter, equation (4) expresses the condition for a pore body of a given size to nucleate (be occupied by vapor). If $\beta$ is assumed constant, larger pores are more likely to nucleate first. Of course this may not necessarily be the case and, in fact, it is more likely that $\beta$ is randomly distributed. However, the assumption, of constant $\beta$ considerably facilitates the calculations, while it does not detract qualitatively from the conclusions to be reached, particularly regarding the growth pattern. Of course, other expressions can also be used (see Parlar and Yortsos (1989)).

In the above, it was also assumed that no bubble detachment due to gravity occurs. $\Lambda$ 
condition for the validity of the latter was obtained by balancing surface and gravity forces, [14]. For the typical values listed in Table 1, we calculate that gravity-induced detachment will not, take place in any pore with size smaller than $\sqrt{\beta} \mu m$.

\section{BUBBLE GROWTH}

Having previously described phase equilibria and nucleation, we may proceed to formulate bubble growth in the pore space. The latter is controlled by surface, inertia, pressure and viscous forces, the driving parameter being the externally imposed superheat in the liquid. For simplicity, we consider first a single vapor "bubble" in an infinitely large porous medium otherwise occupied by liquid (Figure 1). In a later section, the model is extended to consider growth from multiple "bubbles". In the present context, "bubbles" are not spherical, although individual menisci have locally spherical shape.

\subsection{Dimensionless Parameters}

To discuss the growth process, the dimensionless groups that control the growth regimes are, first, introcuced. We follow Szekely and Martins (1971) who studied the growth of a spherical gas bubble in a supersaturated bulk liquid. Typical parameter values correspond to a steam injection process for oil recovery and they are listed in Table 1.

As in Yortsos and Parlar (1989), the variable $\Phi$ expresses the importance of capillarity with respect to supersaturation

$$
\Phi=\frac{2 \gamma H_{0}}{\Delta P}
$$

where $H_{0}$ is a reference mean curvature of the menisci. For the heat transfer problem of interest here, we can alternatively use the imposed superheat $\Delta T$ to get

$$
\Phi=\frac{\Delta T^{* *}}{\Delta T}
$$

where

$$
\Delta T^{* *}=2 \gamma H_{0} \frac{T_{L}^{2}}{\kappa P_{L}}
$$

and

$$
\kappa=\frac{L_{v} M}{R}
$$

Ilere, $L_{v}, M$ and $R$ are latent heat of vaporization, the molecular weight and the ideal gas constant, respectively. For the values in Table $1, \Delta T^{* *}=0.53^{\circ} \mathrm{F}$. Depending upon the 
magnitude of $\Delta P$ (or $\Delta T^{\prime}$ ), growth is described by different regimes. At low superheat values, $\Phi$ is $O(1)$ or larger. (apillary-controlled growth occurs at $\Phi>0(1)$, in which case $\Delta P$ is of the order $2 \gamma H_{0}$. The latter can be significant for small permeabilities.

Another measure of bubble growth is the Jacob number

$$
J a=\frac{\rho_{L} c_{p} \Delta T}{\rho_{V} L_{v}}
$$

whore $\rho_{L}, c_{p}$ and $\rho_{V}$ are liquid density, liquid specific heat capacity and vapor density, respectively. By using the ideal gas law and after some manipulation, the Jacob number can be related to the superheat

$$
J a=\frac{\Delta T}{\Delta T^{*}}
$$

where

$$
\Delta T^{*}=\frac{1}{\rho_{L} c_{p}} \frac{\kappa P_{L}}{T_{L}}
$$

and for the typical values of Table $1, \Delta T^{*}=6.204^{\circ} \mathrm{F}$. This is also inversely proportional to $\Phi$

$$
J a=\frac{\Phi^{*}}{\Phi}
$$

where

$$
\Phi^{*}=\left(2 \gamma H_{0}\right)\left(\rho_{L} c_{p}\right) \frac{T_{L}^{3}}{\left(\kappa P_{L}\right)^{2}}
$$

Clearly, important parameters which control the magnitudes of of $J a$ and $\Phi$ are liquid pressure and temperature in the far field.

Finally, a measure of inertia forces is given by the growth rate parameter

$$
B=\frac{J a^{2}}{\sqrt{G}}
$$

where

$$
G=\frac{R_{0}^{2}}{\rho_{L} \alpha_{L}^{2}} \Delta P
$$

and where $R_{0}$ and $\alpha_{L}$ are initial bubble radius and thermal diffusivity of the liquid, respectively. Following S'zekely and Martins (1971), $B \ll 1$ would imply heat transfer-controlled growth, while $B \gg 1$ implies incrtia-controlled growth, In our subsequent applications, $J a \ll 1$, thus the growth process is heat transfer-controlled only and inertia forces are, therefore, not considered. This is certainly not the case in rapid bubble growth (flash boiling). 


\subsection{Governing Equations}

To express the energy balance in the liquid-occupied space we use the dimensionless notation

$$
\begin{gathered}
\theta=\frac{T_{L}-T_{s a t}}{\Delta T}, \\
\tau=\frac{\alpha_{L} t J a}{R_{0}^{2}} \\
\underline{\xi}=\frac{\underline{x}}{R_{0}}
\end{gathered}
$$

to find

$$
J a\left(\frac{\partial \theta}{\partial \tau}+\underline{\nu_{L}} \cdot \nabla \theta\right)=\nabla^{2} \theta
$$

where

$$
\nu_{L}=\frac{R_{0} u_{L}}{\alpha_{L} J a}
$$

In the above, $\underline{u}_{\underline{L}}, \underline{x}$ and $t$ are liquid velocity, spatial distance and time, respectively, while $T_{s a t}$ corresponds to the far-field liquid pressure. An energy balance at the vapor-liquid interface reads

$$
\nu_{s}=\frac{\partial \theta}{\partial s}
$$

The energy balance in the solid obeys the heat conduction equation. In dimensionless form we have

$$
J a \frac{\partial \Theta}{\partial \tau}=\psi \nabla^{2} \Theta
$$

where

$$
\Theta=\frac{T_{s}-T_{s a t}}{\Delta T}
$$

and $T_{s}$ and $\psi$ are the solid temperature and the ratio of solid to liquid thermal diffusivities, respectively. At the solid-liquid interface, continuity of thermal energy yields

$$
\frac{\partial \theta}{\partial s}=\mu \frac{\partial \Theta}{\partial s}
$$

where $\mu$ is the ratio of solid to liquid conductivities.

At the vapor-liquid interface, and for low superheats, inertia and viscous forces are negligible and the momentum balance simply reads

$$
\Pi=\Phi \eta
$$


where

$$
\Pi=\frac{P_{V}-P_{L}}{\Delta P}
$$

and

$$
\eta=\frac{H}{H_{0}}
$$

where $H$ is the mean curvature. Alternatively, $\Pi$ at the interface can be related to $\theta$ by the Clutusius-Clapeyron equation. We note that at the low Re regime, fluid flow is described by Stokes equation, which is commonly approximated for porous media by a Laplace equation,

$$
\nabla^{2} p=0
$$

Appropriate boundary and initial conditions are

$$
\theta=1 \quad \text { at } \quad \underline{\xi} \rightarrow \infty \quad \text { or } \quad \tau=0
$$

and

$$
\underline{\nu_{L}}=0 \quad \text { at } \quad \underline{\xi} \rightarrow \infty
$$

The problem is, in principle, completely specified by the above. However, a full solution will not be attempted here. Instead, some special, and quite limiting, cases are considered that allow for a simpler description. In all cases described below low superheats are assumed.

\subsection{Low Superheats}

At conditions of low superheats, then $J a \ll 1$, and both balance equations above reduce to the Laplace equation, (provided also that $J a \ll \psi$ ),

$$
\begin{aligned}
& \nabla^{2} \theta=0 \\
& \nabla^{2} \Theta=0
\end{aligned}
$$

At such condixions, the temperature fields in both liquid and solid are quasi-static. This facilitates considerably the analysis. Simple solutions are possible for two cases depending on the magnitude of $\Phi$.

(a) When $\Phi>O(1)$, capillary forces solely control the growth and the process is of the percolation (ordinary or invasion) type, which has been well studied. The rules for the occupancy 
of pores by the vapor phase given in Yortsos and Parlar (1989) can be used with minor modifications. Percolation (ordinary or invasion) growth patterns are applicable to quasi-static fields and the above are valid only if the temperature increase rate is small, to allow for equilibrium to be established. Yortsos and Parlar (1989) developed a constraint for the case of pressure depletion in solution gas-drive systems. It is straightforward to modify their result for the case of boiling in porous media. We obtain the following condition on the heating rate

$$
Q \ll \frac{\Delta T^{*}}{\tau^{*}} J a^{3}
$$

where

$$
\tau^{*}=\frac{\xi_{d}^{2} k}{\phi \alpha_{L}}
$$

and $\xi_{d}, k$ and $\phi$ are the dimensionless correlation length in the percolation process, permeability and porosity, respectively. Taking $J a \sim 10^{-3}$ and substituting $\Delta T^{*}=6.204^{\circ} \mathrm{F}$ in the condition above, gives $Q \ll 32.497^{\circ} \mathrm{F} /$ day for percolation growth patterns to be valid. This constraint is likely to be violated for typical, laboratory scale boiling processes where heating rates are generally high. On the other hand, it is well within the limits of applications involving steam injection for oil recovery or geothermal systems, where processes are slow and equilibrium can be reached.

(b) When $\Phi \ll 1$, capillarity is not important, and in the absence of solid conduction, the process is of tre DLA (Diffusion-Limited-Aggregation) type. In this case, equilibrium states are not possible and there is continous growth until full occupancy if the porous medium by vapor. Clearly, at least for this case, classical concepts such as relative permeabilities need to be reexamined.

\subsection{Moderate and Large Superheats}

Before proceeding we should briefly comment on the case of larger superheats. Now $J a$ is $O(1)$ or larger. Under the condition $B \ll 1$, inertia and viscous forces are still negligible. Therefore, the growth process is heat transfer-controlled, but now the temperature field is no longer quasi-static. This problem is quite complex, requiring for its solution the consideration of both convection and unsteady state terms. Finally, at very large superheat, $J a \gg 1$, while also $B \gg 1$, implying that inertia controlls growth. This is the case of explosive growth, which is out side the present scope. 
The above pertained to growth of a single "bubble" in porous media. In the remaining, we shall consider growth of "bubbles" from multiple sites, as a result of the activation of many nucleation sites. Growth from multiple sites was studied by Yortsos and Parlar (1989) who modified ordinary percolation in infinite Bethe lattices. Their results are in closed-form expressions. Here, we present numerical results for the boiling case by considering the same percolation problem but in a square lattice. The model in [14] was developed for infinitesystems, where there is no depletion of supersaturation. Here, we shall also consider the adiabatic case, where the available energy is finite.

We should point out that an important difference with the solution gas-drive problem is the participation of the solid matrix to transport. In general, three different regimes can be distinguished according to the ratio of conductivities $\left(\mu=\lambda_{s} / \lambda_{L}\right)$ :

$\mu \ll 1 \rightarrow$ where only the liquid is conducting

$\mu \sim 1 \rightarrow$ where both solid and liquid participate in the conduction

$\mu \gg 1 \rightarrow$ where only the solid is conducting

Each of these three cases finds many applications. The solution gas-drive model in Yortsos and Parlar (1989) pertains to the first limit, where the solid conductivity is small and its participation to heat transfer is negligible. Many boiling applications in porous media lie closer to the third limit, with the ratio $\mu$ as high as 10 . To account for this, a variant of gradient percolation is developed.

\section{PERCOLATION MODELS}

We consider bubble growth from multiple nucleation sites corresponding to low superheats (or to $\Phi \sim 1$ ), where the growth process is of the percolation type. In this case, infinitesimally small $\Delta T$ steps corresponding to a slow heating rate are imposed, such that the supersaturation is always balanced by capillary pressure. Three cases are considered: The first case is slow, uniform heating at an increased superheat, the second corresponds to adiabatic expansion, although at spatially uniform temperature and pressure, while the third involves static vaporliquid distributions in the presence of a temperature gradient. Main objectives are to find the distributions of the vapor and liquid phases in the pore space, for each process, so that classical continuum concepts, such as relative permeabilities, can be calculated. This is, by no means, a trivial exercise. As previously implied, the present models mostly address effects of nucleation 
on these properties.

We use both bond and site distributions. The rules for the activation of the nucleation sites and the occupancy of pore space by the vapor are simple. Given a superheat $\Delta T=T_{L}-T_{\text {sat }}$, pores with throat radius

$$
r_{b} \geq R^{*}=\frac{2 \gamma T_{s a t}}{\rho_{V} L_{v} \Delta T}
$$

are occupied by the vapor phase if they are already connected to vapor-occupied sites or to nucleation sites. New nucleation sites are activated if the host pore bodies have a large enough radius

$$
r_{s} \geq \frac{R^{*}}{\beta}
$$

An important difference between this model and ordinary percolation is nucleation. Classical percolation is recovered in the limit $\beta=1$.

\subsection{Ordinary Percolation with Nucleation}

Simulation results are shown in Figure 6, for square $(30 \times 30)$ lattice network at three different stages of growth corresponding to increasing superheats. Pore body and throat sizes were randomly assigned from a uniform distribution. In Figure 6, gray denotes vapor-occupied sites, black denotes nucleation sites and white denotes liquid-occupied sites. It is apparent that all vapor-occupied pores are connected to a nucleation site. In this model of unlimited supply of supersaturation, more nucleation sites are activated as the supersaturation increases. However, not all nucleation sites have expanded, particularly at low superheats. Since no temperature gradients are considered, no frontal structures (spatial gradients) develop. The predictions from this model are similar to those from the analytical study of Yortsos and Parlar (1989). In particular, a critical supersaturation exists for the onset of bulk vapor flow, and relative permeabilities can be calculated as a function of the nucleation parameter $\beta$. We refer the reader to [14] for details. Figure 7 shows relative permeability results reprinted from [14].

Since the process is of the percolation type, the onset of bulk vapor flow occurs when an "infinite" cluster forms, corresponding to the supersaturation

$$
\Delta P_{c}=\frac{2_{r}}{r_{d c}}
$$

where $r_{d c}$ solves

$$
\int_{r_{d c}}^{\infty} \alpha_{b}(r) d r=p_{c}
$$


Here, $\alpha_{b}(r)$ is the bond size distribution, and $p_{c}$ is the percolation threshold. The critical vapor saturation $S_{g c}$ is obtained by adding to the "infinite cluster" the contribution of vapor-occupied sites that are connected to nucleation sites but do not belong to the percolation cluster. It is apparent that the latter value would increase as nucleation is more facilitated. Equivalently, the relative permeability to vapor would decrease as the nucleation fraction increases. These

features are clearly evident in Figure 7. We should point out that when the ratio $i_{i}=\frac{r_{d a}}{r_{s} \text { max }}$ is not zero it is possible that although sufficient superheat is available for bond percolation, vapor occupancy has not yet occured, because of lack of nucleation sites $(\beta<\kappa)$. Percolation then takes place when the first site is nucleated, with superheat corresponding to the radius $\beta r_{s, \text { max }}$, thus resulting into large values of $S_{g c}$. Figure 8 taken from [14] shows this dependence.

Conditions for the validity of this model were developed above. As pointed out, this process is a slow, spatially uniform heating of the liquid in the porous medium. Clearly, this is only an approximation to the actual process. One possible modification regards the depletion of superheat.

\subsection{Percolation with Superheat Depletion}

To model this process, we have simulated vapor growth by pressure reduction in an adiabatic system. Contrary to the previous, the available superheat here is finite. Since vapor expands and displaces liquid which leaves the system, the available amount of initial entalphy is constantly reduced. Further growth can thus occur only by further pressure depletion. We must note that although supeerheat is depleted, competition for growth anong the various bubbles is still absent, because of the assumption of uniform temperatures. Results are shown in Figure 9 for conditions identical to the previous. At th: end of the process, the pressure has been reduced from 202.8 psi to 185 psi, while the temperature reduction is somewhat less, from $383^{\circ} F$ to $375.9^{\circ} \mathrm{F}$. Although the amount of supersaturation or superheat changes, one can nevertheless show that the growth pattern still remains identical to the previous percolation type. This is to be expected, since spatial gradients are not included. One concludes that consideration of finite volume and adiabatic conditions does not change the previous results as far as flow parameters (e.g. relative permeabilities, etc.) is concerned.

Both of the above were modifications of ordinary percolation, corresponding to the case where solid matrix participation is of no relevance. The last section considers gradient perco- 
lation which involves mainly heat conduction by the solid.

\subsection{Gradient Percolation with Nucleation}

A final modification involves the ase where a fixed temperature gradient is assumed across Whe sample. The assumption is that solid conduction dominates the heat transfer $(\mu \gg 1)$,

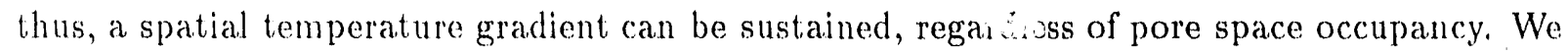
make use of the previous rules for vapor occupancy of a site, for both nucleation and growth. Note, however, that fluid flow, particularly countercurrent flow, is not considered. This is a substantial limitation: A temperature gradient in the vapor phase requires a corresponding pressure gradient, thus vapor flow. At steady state, this must be counterbalanced by liquid flow in the opposite direction. We are currently investingating the effects of this approximation in a study of heat pipes using pore network models.

Allowing for a spatial gradient gives rise to gradient percolation. This concept has been introduced in recent years as a variation of classical percolation. It is recalled that in ordinary percolation the probability for the occupation of a site is spatially uniform. For gradient percolation, on the other hand, it is given by a specified function which is variable in space. This difference substantially changes the pattern of growth from that of classical percolation. In particular, fronts can be delineated.

Gouyet et al. (1985) introduced gradient percolation in their study of diffusion in solids. They used the complementary crror function to describe the probability distribution in space, for two-dimensional, square and triangular lattice networks. Using certain clustering and occupation rules, they examined the nature of the diffusion front created. They found out that this front is fractal (with dimension 1.75), while the probability value at the mean position of this front is related to the percolation threshold of ordinary percolation. In a subsequent study of 3-d cubic lattices, (Conyet et al. 1986) they observed significant differences between 2-d and 3-d simulations. In particular, the shape of the density profile at the frontier changed. Furthermore, the probability density value at the mean frontier position was different than $p_{c}$. Nevertheless, many scaling properties were found (Rosso et al. 1.988).

Concerning bubble growth in porous media, when solid conduction predominates $(\mu \gg 1)$. the application of gradient percolation is appealing. At such conditions, the temperature field is clecoupled from the occupancy scheme, which it actually dictates. For application to boiling, 
i'e have modified gradient percolation by adding bond statistics and nucleation effects. We consider uniform size distributions for sites and bonds, and a lincar temperature profile. The paraneters affecting the occupancy are pore and bond size distributions, the values of $\beta$ and the imposed gradient $\Delta T$. Growth patterns for modified gradient percolation models are shown in ligure 10 for two different values of the nucleation parameter $\beta$. Gray denotes vapor-occupied sites, while black denotes the interface which separates the infinite clusters of liquid-occupied sites and vapor-occupied sites. As $\beta$ increases, more nucleation siles are available and this results in higher occupancy of pores by vapor. This increase in $\beta$ shifts the interface toward the liquid side. Comparison between Figures 6 and 10 shows that the two patterns are quite different. A steam front is clearly distinguishable in the gradient percolation case. However, when spatial gradients are involved the definition of continuum quantities becomes problematic. Further progress requires additional research which is currently in progress in our laboratory.

\section{CONCLUSIONS}

In this paper we applied the general methodology developed by Yortsos and Parlar (1989) for solution gas-drive processes, to the case of boiling in porous media. Phase equilibria and stability, nucleation, pore level microstructure and bubble growth were considered. At conditions of low superheat where the temperature field is quasi-static, the growth problem was shown to be similar to a drainage displacement process, with the addition of growth :om activated nucleation sites. The case of uniform heating was described using a percolation approach, under the constraint that the heating rate must be significantly lower than $32.49^{\circ} \mathrm{F} / \mathrm{day}$. Field cases relevent to steam injection or geothermal processes may obey this constraint. If such conditions prevail, relative permeability functions and critical saturation values can be employed in a modified form to account for nucleation. To account for temperature gradients in case where conduction in the solid dominates the heat transfer, and in the absence of flow, we have modified gradient percolation. Although an improvement over the uniform percolation model, these modifications are still tentative. We are currently involved in an effort to resolve many of the important issues left unanswered, including the effect of the rate of superheat and the competition between nucleation sites for growth. 


\section{ACKNOWLEDGEMENTS}

'This work was partly supported by DOE contract DE-FG19-87BC14126, the contribution of which is gratefully acknowledged. 


\section{REFERENCES}

1. G. Schubert and J.M. Straus, Steam-water counterflow in porous media, J. Geophysical Res. 84, 16211979.

2. M. Prats, Thermal Recovery, SPE Monograph, Vol, 7, Dallas, Texas (1982).

3. C. Doughty and K. Pruess, A semianalytical solution for heat-pipe effects near high-level nuclear waste packages burried in partially saturated geological media, Int. J. Heat Mass Transfer 31, 79-90 (1988).

4. H.H. Bau and K.E. Torrance, Boiling in low-permeability porous materials, Int. J. Heat Mass Transfer 25, 45-54 (1982).

5. L.E. Scriven, On the uynamics of phase growth, Chem. Eng. Sci. 10, 1-13 (1959).

6. M.S. Plesset and S.A. Zwick, The growth of vapor bubbles in superheated liquids, J. Appl. Phys. 25, 493-500 (1954).

7. M.S. Plesset and A. Prosperetti, Bubble dynamics anad cavitation, Ann. Rev. Fluid Mech. 9, 145-185 (1977).

8. S.L. Solov'yev and S.A. Kovalev, Heat transfer in the evaporation of a liquid on a porous surface, Tepl. Vysok. Temp., 22(3), 528-536 (1984).

9. M.A. Styrikovich, S.P. Malyshenko, A.B. Andrianov and I.V. Talaev, Investigation of boiling on porous surfaces, Heat Transfer - Sov. Res., 11(1), 23-29 (1987).

10. S.A. Kovalev, S.L. Solov'yev and O.A. Ovodkov, Liquid boiling on porous surfaces, Heat Transfer - Sov. Res., 19(3), 109-120(1987).

11. K.S. Udell, Heat transfer in porous media heated from above with evaporation, condensation, capillary effects, J. Heat Trans. 105, 485-495 (1983).

12. M. Parlar and Y.C. Yortsos, Percolation theory of steam-water relative permeability, paper SPE 16969 presented at the 62nd Annual Meeting of SPE, Dallas, TX, Sept., 27-30) (1987). 
13. J.M. Sanchez and R.S. Schechter, paper SPE 16967 presented at the 62nd Annual SPL Fall Meeting, Dallas, TX, Sept. 27-30 (1987).

14. Y.C. Yortsos and M. Parlar, Phase change in porous media: Application to solution gas drive, paper SPE 19697 presented at the 64th Annual Technical Conference and Exhibition of SPE, San Antonio, TX, Oct., 8-11 (1989).

15. C. Satik, M. Parlar and Y.C. Yortsos, A study of steady-state steam-water counterflow in porous media, Int. J.'Heat Mass Transfer, in press (1991).

16. G.J. Hirasaki, D.J. O'Meara Jr. and J.A. Rohan, Centrifuge measurements of capillary pressure: Part 2 - cavitation, paper SPE 18592 presented at the 63rd Annual Meeting o1 SPE, Houston, TX, Oct. 2-5, 1988.

17. T.W. Forest, The stability of gaseous nuclei at liquid-solid interfaces, J. Appl. Phys, 53 (1982) $6191-6201$.

18. M. Parlar and Y.C. Yortsos, Nucleation and pore geometry effects on capillary desportion processes in porous media, J. Colloid Interface Sci. 132, 425 (1989).

19. J. Szekely and G.P. Martins, Non-equilibrium effects in the growth of spherical gas bubbles due to solute diffusion, Chem. Eng. Sci. 26, 147-159 (1971).

20. J.F. Gouyet, M. Rosso and B. Sapoval, Determination of percolation probability from the use of a concentration gradient, Phys. Rev. B, 32: 6053 (1985).

21. M. Rosso, J.F. Gouyet and B. Sapoval, Gradient percolation in three dimensions and relation to diffusion fronts, Phys. Rev. B 57, 3195 (1986).

22. J.F. Gonyet, M. Rosso and B. Sapoval, Fractal structure in diffusion and invasion fronts in three-dimensional lattices through the gradient percolation approach, Phys. Rev. B $37,1832(1988)$. 
Table 1: Typical Values of Parameters.

$$
\begin{aligned}
& c_{p}=1.059 \mathrm{Btu} / \mathrm{lbm} /{ }^{\circ} \mathrm{F} \\
& H_{o}^{-1}=15 \mu \mathrm{m} \\
& k=1 \text { darcy } \\
& L_{v}=841.75 \mathrm{Btu} / \mathrm{lbm} \\
& M=18 \mathrm{lb} / \mathrm{mol} \\
& P_{L}=202.819 \mathrm{psi} \\
& T_{L}=383^{\circ} \mathrm{F} \\
& \alpha_{L}=6.7113 * 10^{-3} \mathrm{ft}^{2} / \mathrm{h} \\
& \gamma=60.0 \mathrm{dymles} / \mathrm{cm}^{2} \\
& \lambda_{L}=0.386 \mathrm{Btu} / \mathrm{h}^{2} / \mathrm{ft}^{\circ}{ }^{\circ} \mathrm{F} \\
& \xi_{d}=1 \\
& \rho_{L}=54.31 \mathrm{lbm} / \mathrm{ft}^{3} \\
& \phi=0.35
\end{aligned}
$$




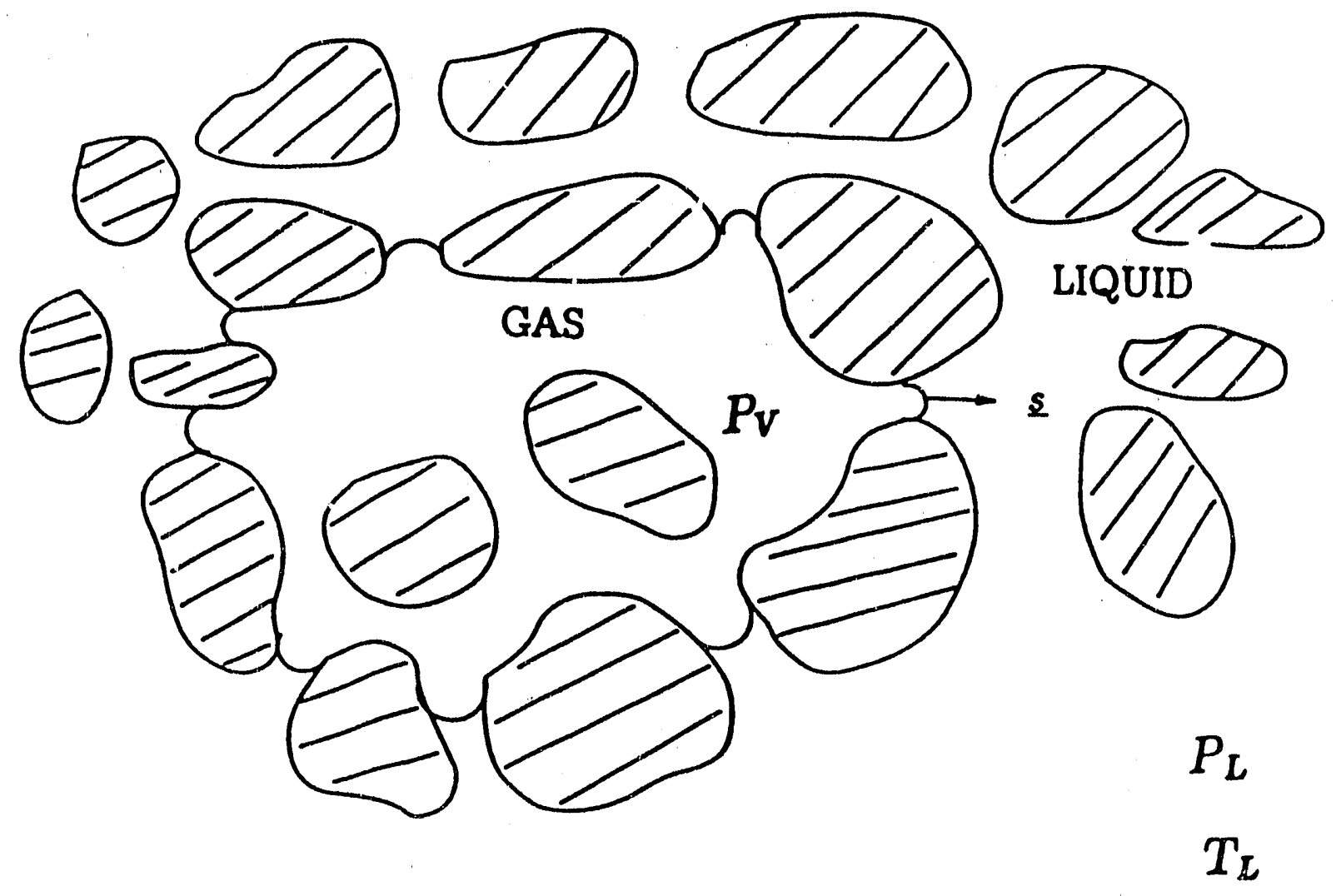

Figure 1: Schematic of a "Bubble" in a Porous Medium. 


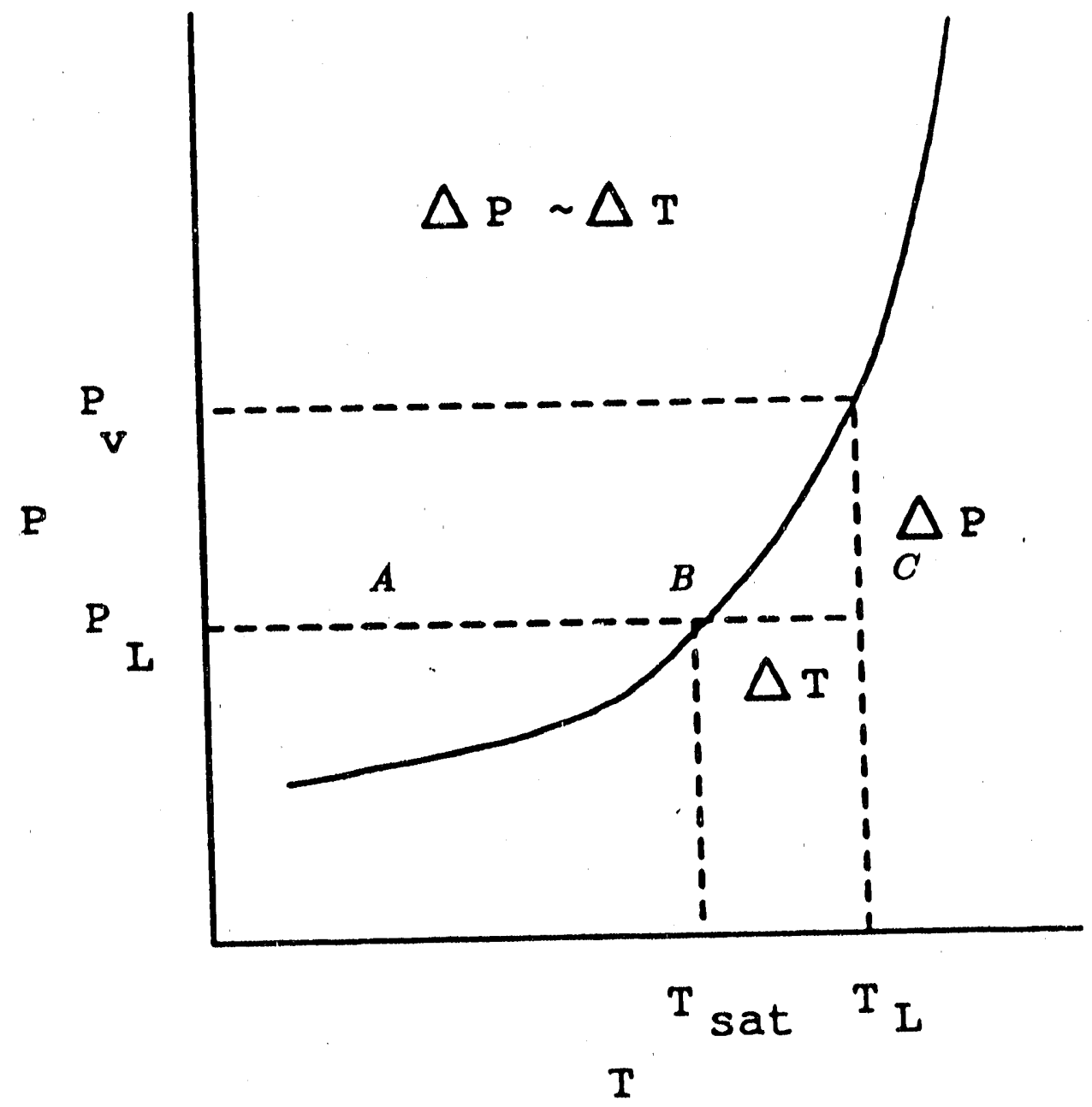

Figure 2: Schematic of Equilibrium and Supersaturations. 


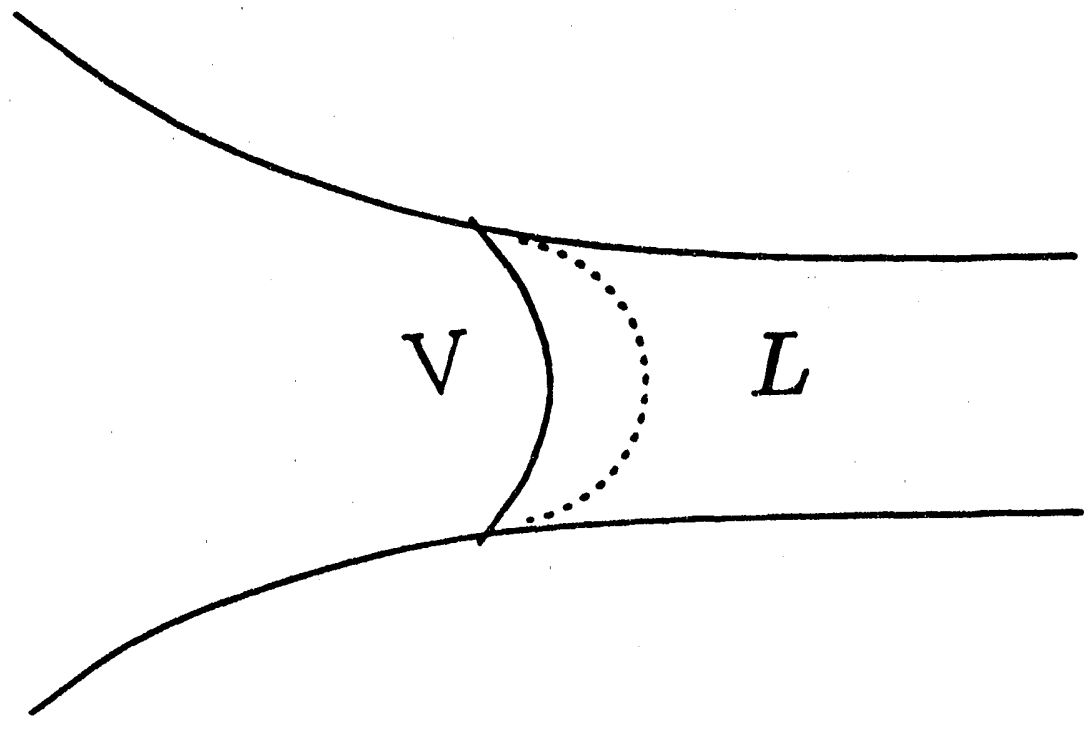

Figure 3: Stability of a Vapor-Liquid Interface in a Converging Geometry. 


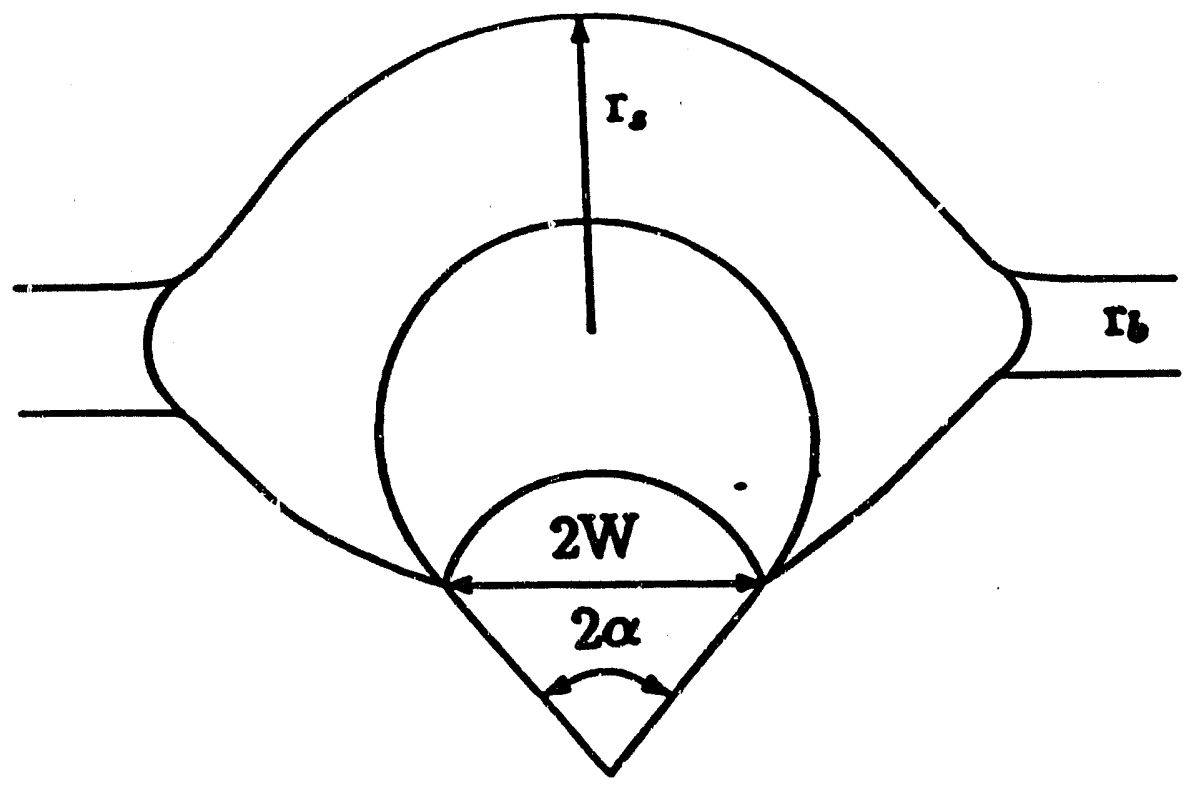

Figure 4: Schematic of a Nucleation Site in a Pore Body. 


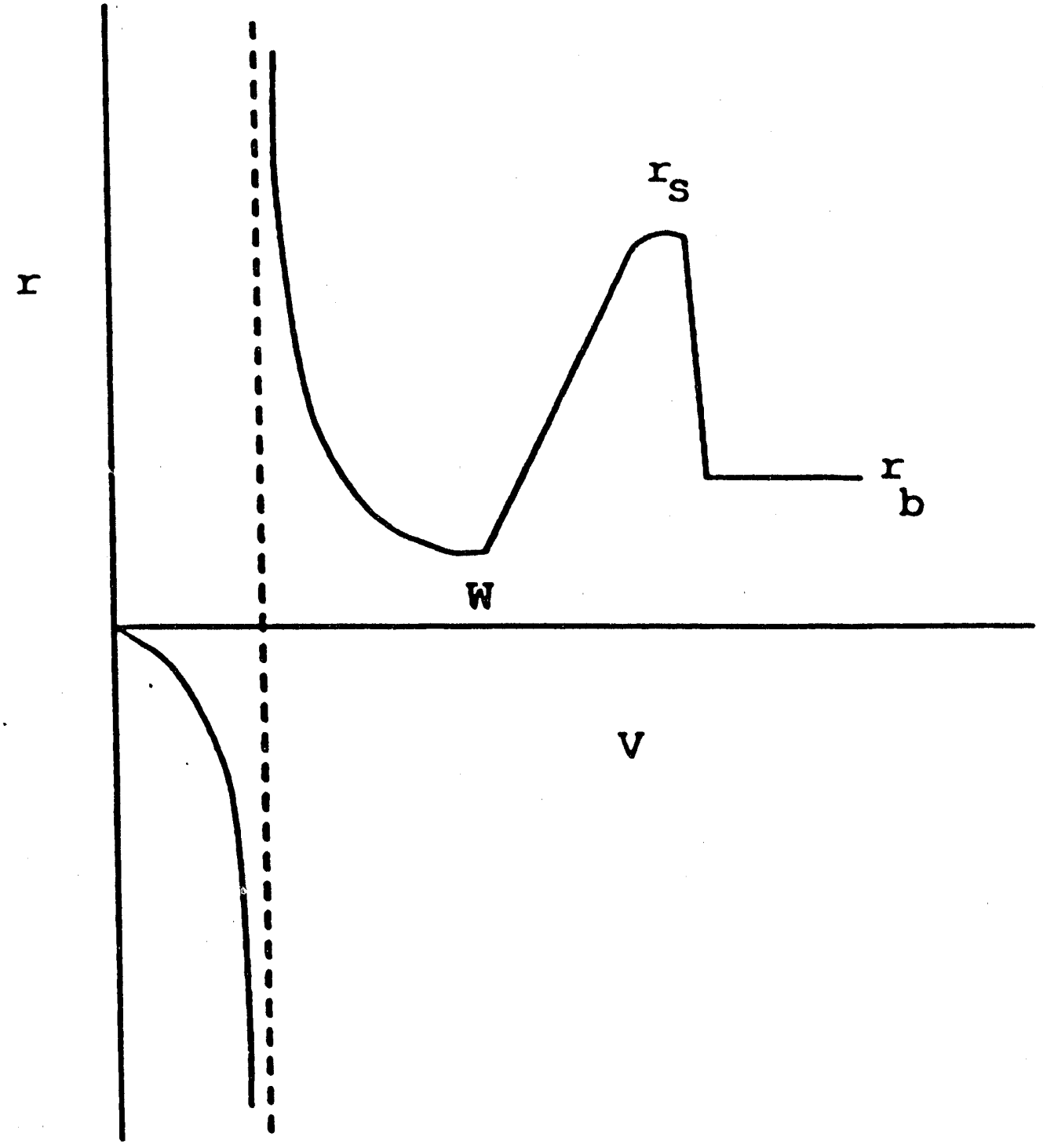

Figure 5: Bubble Radius-Volume Equilibrium for the Hydrophobic Cavity of Fig. 4. 

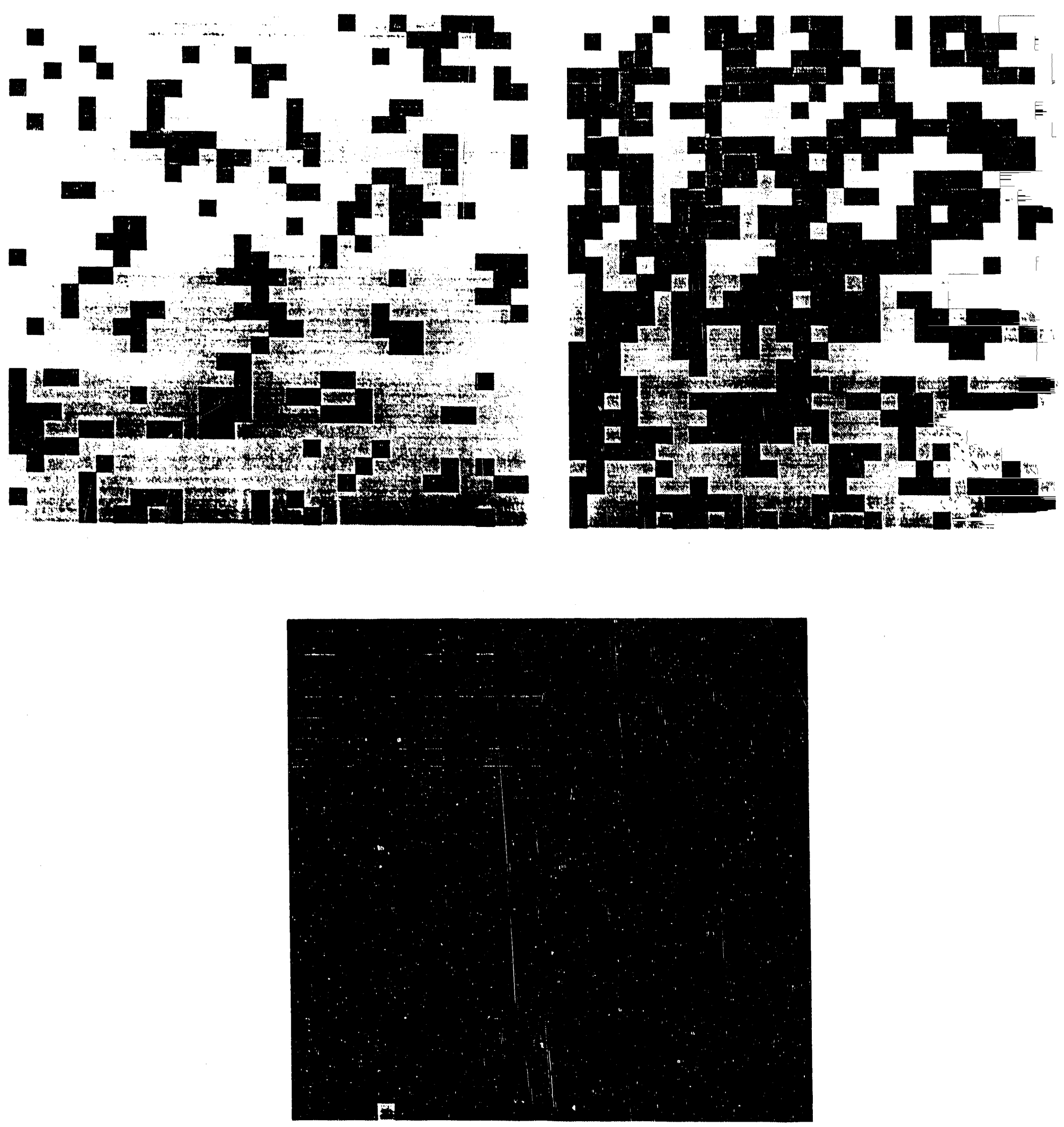

Figure 6: Bubble Growth in Percolation from Nucleation Sites in a Square Lattice at Three Different Stages of Growth ( $p=0.221,0.555$ and 0.9988 , respectively). 


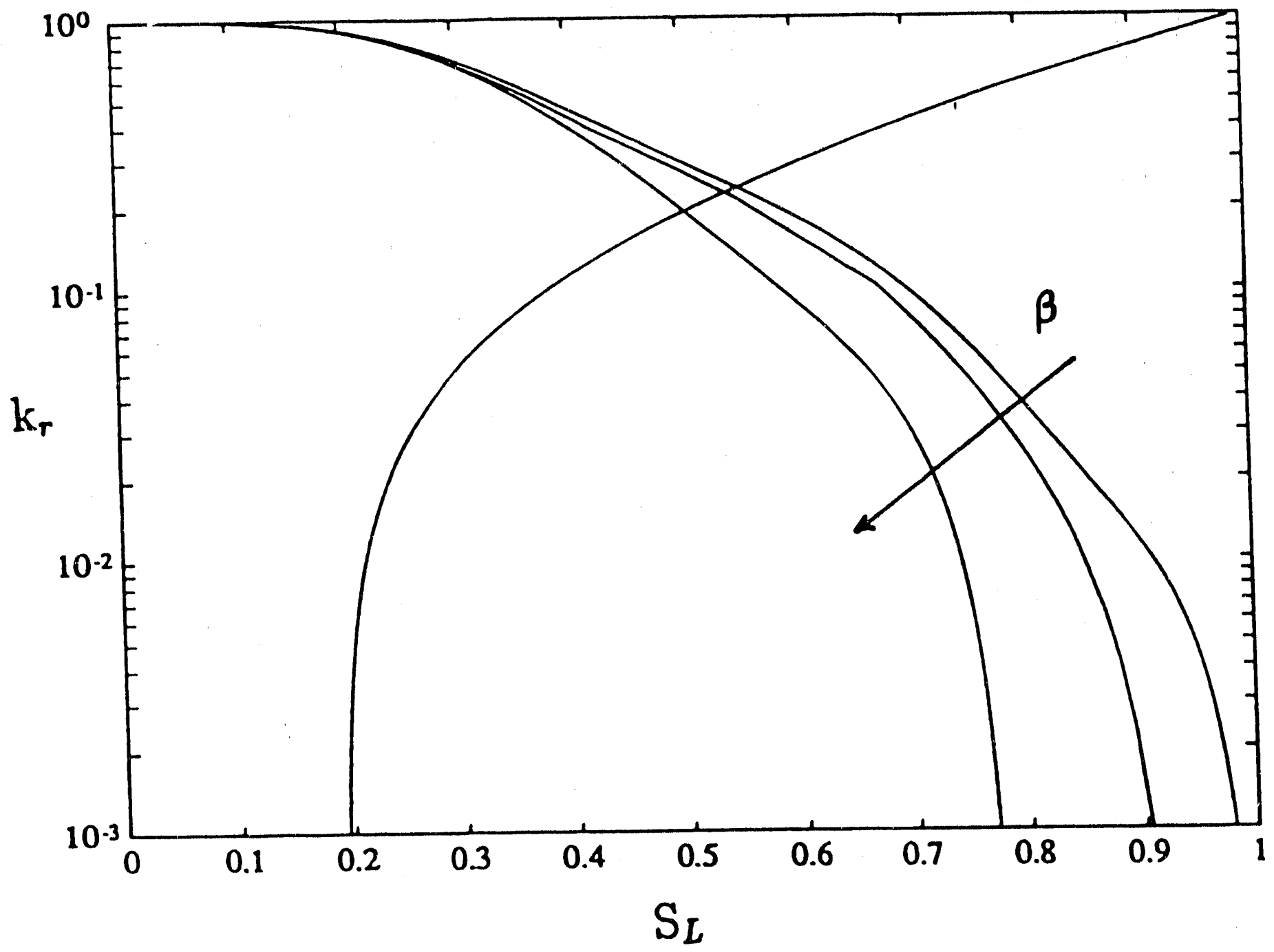

Figure 7: Relative Permeabilities as a Function of $\beta$ (from [14]). 


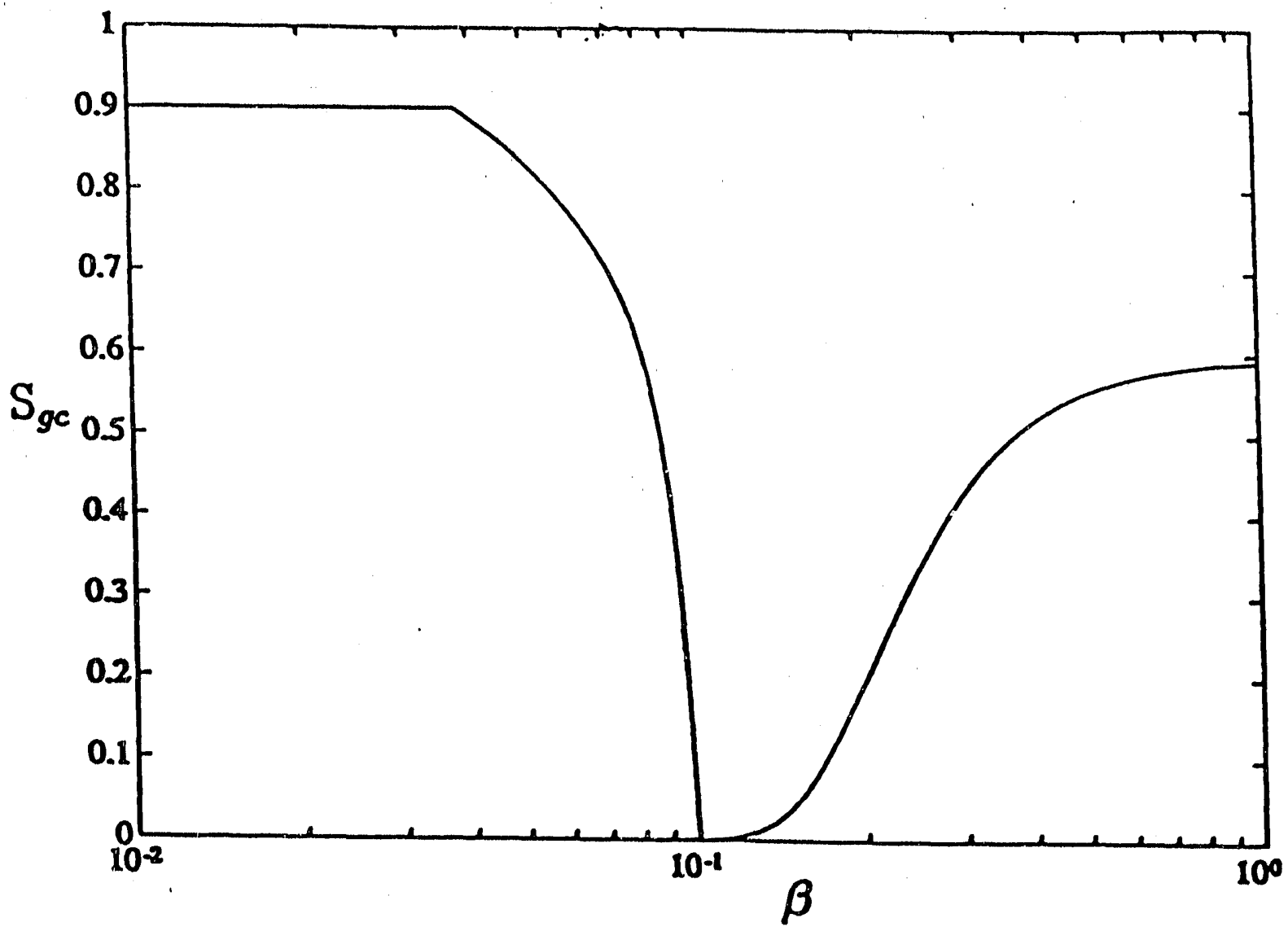

Figure 8: Critical Gas Saturation as a Function of $\beta$ (from [14], $\kappa=0.1$ ). 


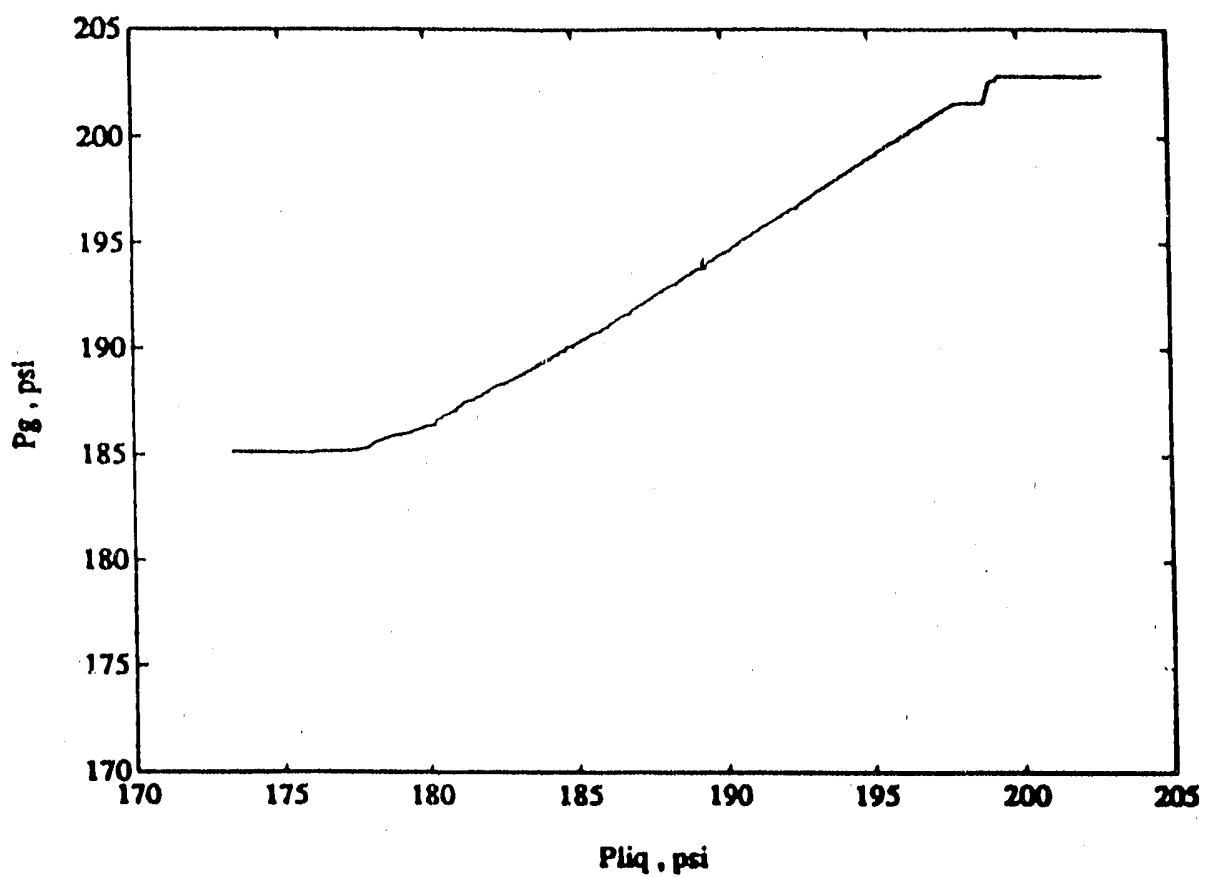

(a)

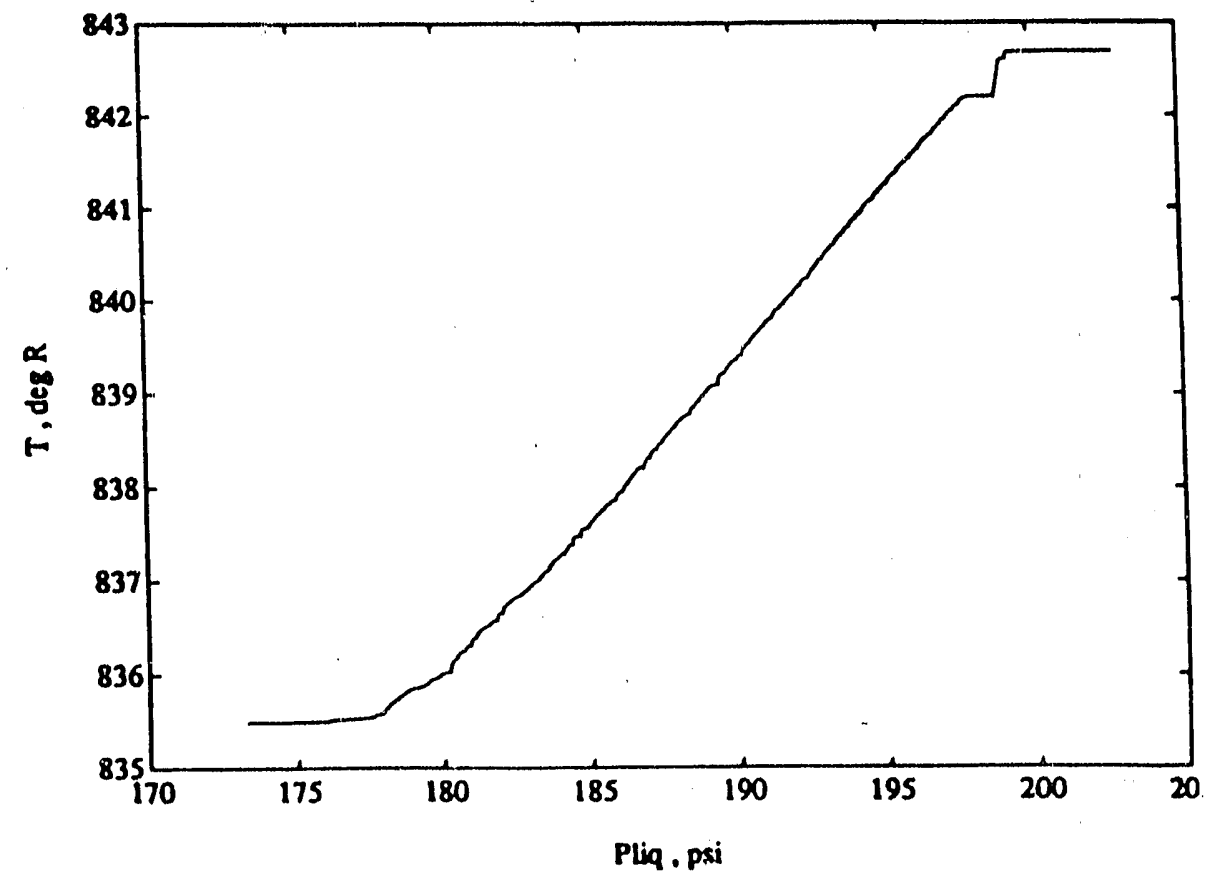

(b)

Figure 9: Supersaturation Paths for Adiabatic Depletion: (a) Pressurı, (b) Temperature. 


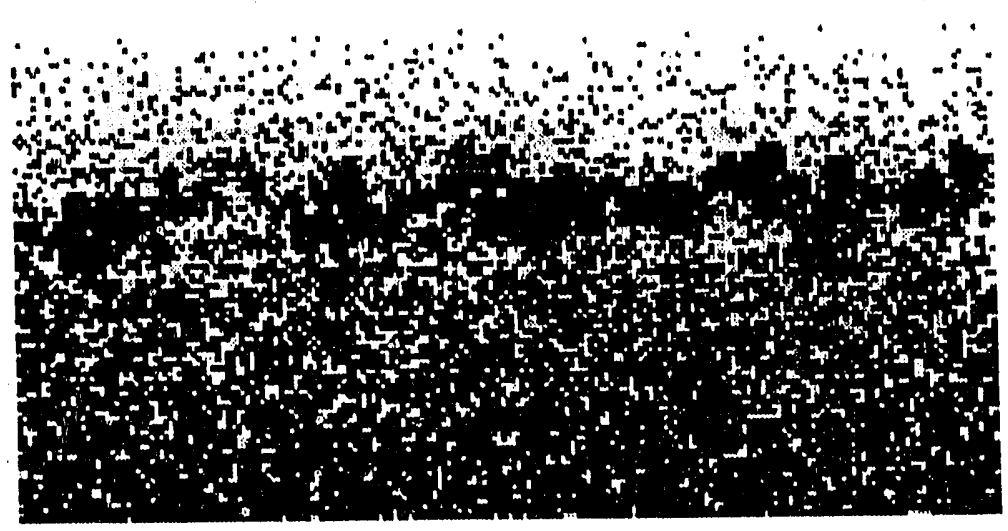

(a)

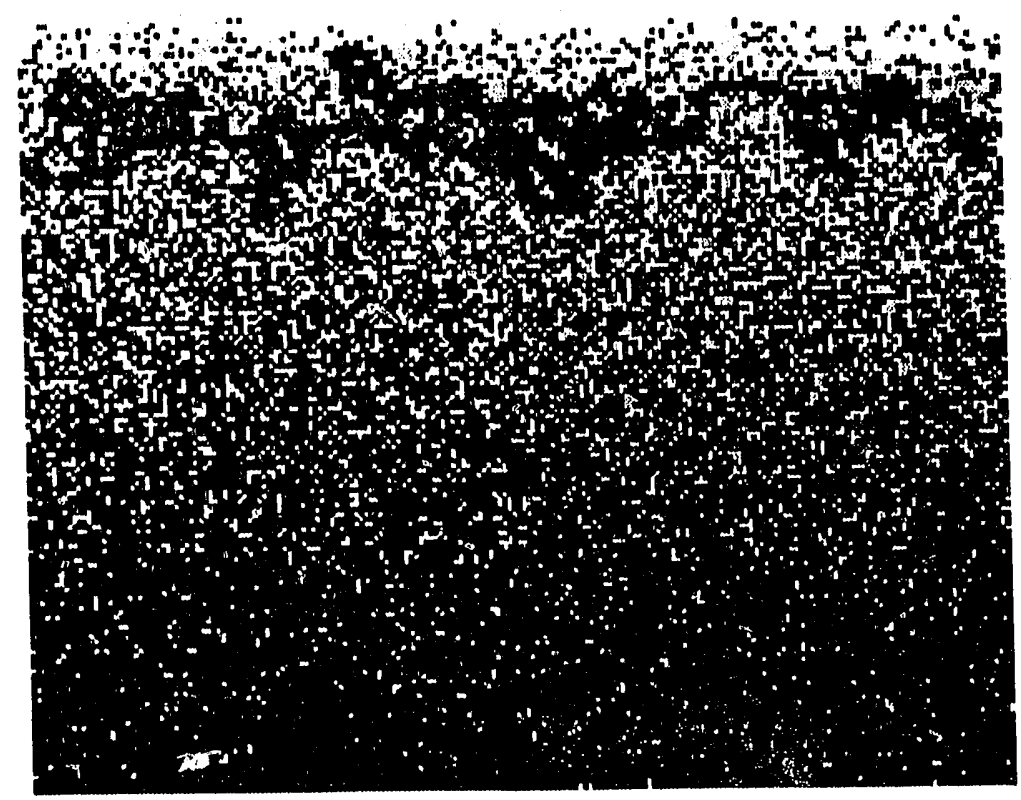

(b)

Figire: 10: Vapor-Liquid Equilibrium in a Temperature Gradient Using Gradient Percolation for Different Values of $\beta$ : (a) $\beta=0.2$, (b) $\beta=0.5$. 

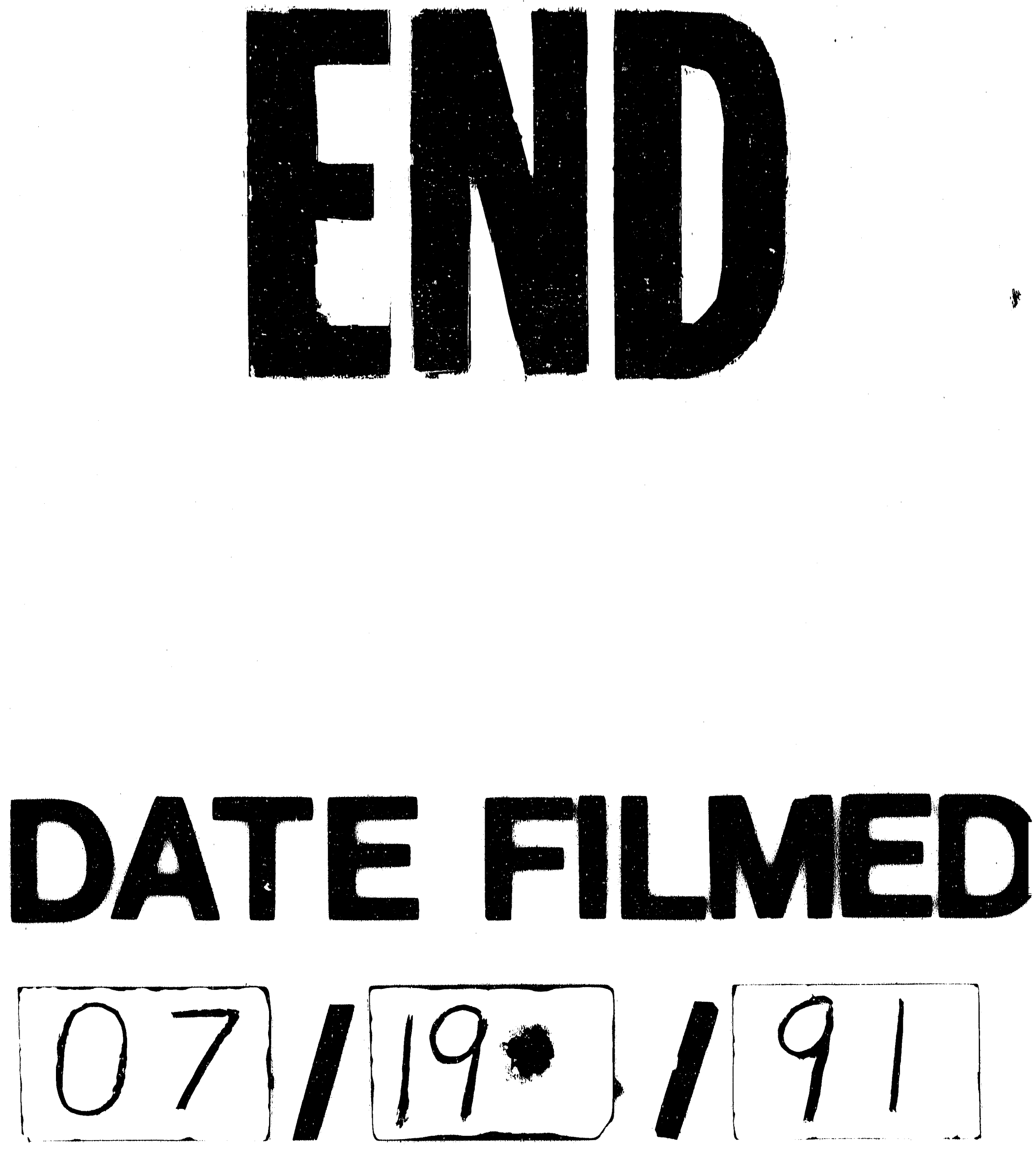


$$
\bar{c}
$$

